PROCEEDINGS OF THE

AMERICAN MATHEMATICAL SOCIETY

Volume 133, Number 9, Pages 2751-2759

S 0002-9939(05)07789-0

Article electronically published on April 19, 2005

\title{
PLANAR ALGEBRAS AND THE OCNEANU-SZYMAŃSKI THEOREM
}

\author{
PARAMITA DAS AND VIJAY KODIYALAM
}

(Communicated by David R. Larson)

\begin{abstract}
We give a very simple 'planar algebra' proof of the part of the Ocneanu-Szymański theorem which asserts that for a finite index, depth two, irreducible $I I_{1}$-subfactor $N \subset M$, the relative commutants $N^{\prime} \cap M_{1}$ and $M^{\prime} \cap$ $M_{2}$ admit mutually dual Kac algebra structures. In the hyperfinite case, the same techniques also prove the other part, which asserts that $N^{\prime} \cap M_{1}$ acts on $M$ with invariants $N$.
\end{abstract}

The Ocneanu-Szymański theorem $\mathrm{Cnn}$, $[\mathrm{Szy}$ is a basic result that has inspired interesting generalisations of Hopf and Kac algebras BhmNllSzl, NksVnr. Given the plethora of algebraic structures inherent in the planar algebras of [Jns], it is natural to expect relationships between the planar algebra and the Kac algebra structures. These are what we elucidate in this paper for the case of an irreducible, depth two subfactor. The reducible case is treated in [Das].

\section{Planar algebras}

We begin with a brief summary of planar algebras. For details, the reader is referred to Jns or to KdyLndSnd. The basic structure that underlies planar algebras is an action by the 'coloured operad of planar tangles', which concept we will now explain. Consider the set $\mathrm{Col}=\left\{0_{+}, 0_{-}, 1,2, \cdots\right\}$, elements of which we call colours. We will not define a tangle here, but several examples are shown in Figure 1. We point out some of their features.

1.1. Tangles. Each tangle has an external box, denoted $D_{0}$, and some (possibly 0 ) ordered internal boxes denoted $D_{1}, D_{2}, \cdots$. Each box has an even number of points marked on its boundary (again possibly 0 ) - a box with $2 k$ points on its boundary is called a $k$-box.

If a box has at least one point marked on its boundary, one of them is distinguished and marked with a ' $*$ '. There is also given a collection of disjoint curves, each of which is either closed or joins a marked point on one of the boxes to another such point. The whole picture is to be planar, and each marked point on a box must be the end-point of one of the curves. Finally, there is given a black-and-white shading of the regions, such that moving away from (resp. towards) the $*$ on one of the internal boxes (resp. the external box) along the curve of which it is the end-point, a black region is to the right. A 0-box is said to be a $0_{+}$-box if the

Received by the editors December 2, 2002 and, in revised form, June 25, 2003.

1991 Mathematics Subject Classification. Primary 54C40, 14E20; Secondary 46E25, 20C20.

Key words and phrases. Planar algebra, subfactor, Kac algebra, Ocneanu-Szymański theorem.

(C)2005 American Mathematical Society 2751

Reverts to public domain 28 years from publication 


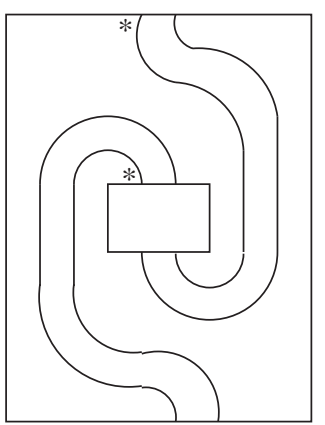

$R=R_{2}^{2}$

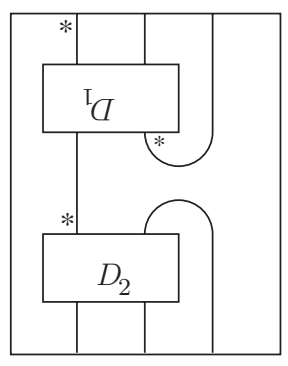

$W=W_{2,2}^{3}$

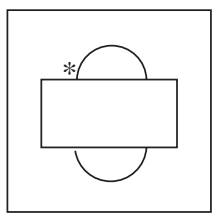

$L=L_{2}^{0_{+}}$

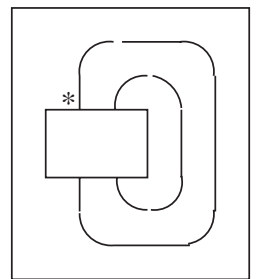

$t r_{2}^{0_{+}}$

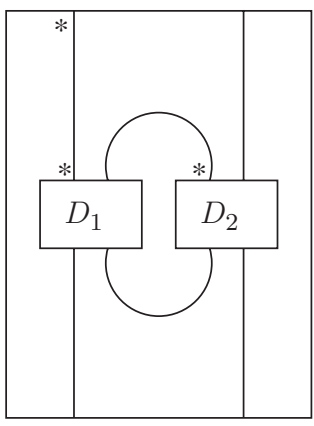

$C=C_{2,2}^{2}$

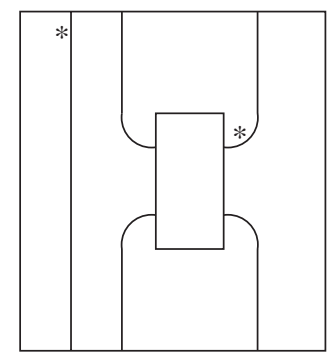

$F=F_{2}^{3}$

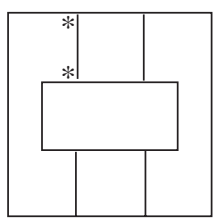

$I_{2}^{2}$

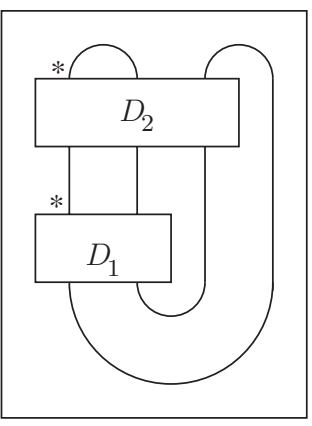

$$
B=B_{2,3}^{0+}
$$

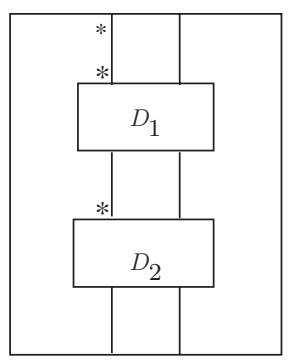

$M_{2,2}^{2}$

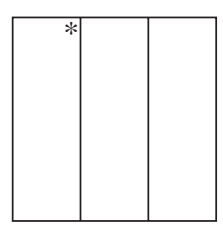

$1^{2}$

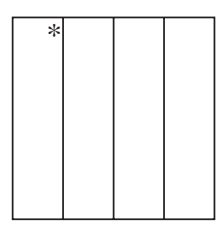

$1^{3}$

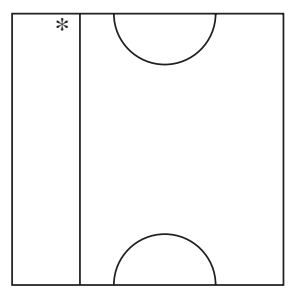

$\mathcal{E}^{3}$

Figure 1. Some useful tangles

region touching its boundary is white and a $0_{-}$-box otherwise. A tangle is said to be a $k$-tangle if its external box is of colour $k$. As a matter of notation, tangles will be given names, including subscripts and a superscript. The subscripts indicate 
the colours of the internal boxes and the superscript indicates the colour of the tangle. Given the superscript, the entire shading scheme is determined and will not be indicated. We will not distinguish between tangles that can be obtained from each other by a planar isotopy preserving the $*$ 's, the shading and the ordering of the internal boxes.

1.2. Operad of planar tangles. The basic operation that one can perform on tangles is substitution of one into a box of another. If $T$ is a tangle that has some internal boxes $D_{i_{1}}, \cdots, D_{i_{j}}$ of colours $k_{i_{1}}, \cdots, k_{i_{j}}$ and if $S_{1}, \cdots, S_{j}$ are arbitrary tangles of colours $k_{i_{1}}, \cdots, k_{i_{j}}$, then we may substitute $S_{t}$ into the box $D_{i_{t}}$ of $T$ for each $t$, such that the ' $*$ 's match', to get a new tangle that will be denoted $T \circ_{\left(D_{i_{1}}, \cdots, D_{i_{j}}\right)}\left(S_{1}, \cdots, S_{j}\right)$. If $T$ has a single internal box, the subscript to $\circ$ will be omitted and indeed, the box $D_{1}$ itself will not be named, as is clear from Figure 1 The collection of tangles along with the substitution operation is called the coloured operad of planar tangles.

1.3. Planar algebras. A planar algebra $P$ is an algebra over the coloured operad of planar tangles. By this we mean the following: $P$ is a collection $\left\{P_{k}\right\}$ of vector spaces for $k \in \mathrm{Col}$ and maps $Z_{T}: P_{k_{1}} \otimes P_{k_{2}} \otimes \cdots \otimes P_{k_{b}} \rightarrow P_{k_{0}}$ for each $k_{0^{-}}$ tangle $T$ with internal boxes of colours $k_{1}, k_{2}, \cdots, k_{b}$. The collection of maps is to be 'compatible with substitution of tangles and renumbering of internal boxes' in an obvious manner. Further, planar algebras are required to be non-degenerate in the sense that for each $k \in C o l$, the map $Z_{I_{k}^{k}}$ is the identity map of $P_{k}$. A pleasant verification then shows that each $P_{k}$ has the structure of an associative, unital algebra, where the multiplication is given by $Z_{M_{k, k}^{k}}$ and the unit by $Z_{1^{k}}(1)$, which we will denote by $\mathbb{1}_{k}$. The tangles $I_{k}^{k}, M_{k, k}^{k}$ and $1^{k}$ are the straightforward generalisations to $k$-tangles of the tangles $I_{2}^{2}, M_{2,2}^{2}$ and $1^{2}$ that appear in Figure 1

1.4. Subfactor planar algebras. Among planar algebras, the ones that we will be interested in are the subfactor planar algebras. These are finite dimensional and connected in the sense that each $P_{k}$ is a finite-dimensional vector space and $P_{0_{ \pm}}$ are one dimensional. They have a positive modulus $\delta$, meaning that closed loops in a tangle $T$ contribute a multiplicative factor of $\delta$ in $Z_{T}$. They are spherical in that for a 0 -tangle $T$, the function $Z_{T}$ is not just planar isotopy invariant but also an isotopy invariant of the tangle regarded as embedded on the surface of the two sphere. Further, each $P_{k}$ is a $C^{*}$-algebra in such a way that for a $k_{0}$-tangle $T$ with internal boxes of colours $k_{1}, k_{2}, \cdots, k_{b}$ and $x_{i} \in P_{k_{i}}$,

$$
Z_{T}\left(x_{1} \otimes \cdots \otimes x_{b}\right)^{*}=Z_{T^{*}}\left(x_{1}^{*} \otimes \cdots \otimes x_{b}^{*}\right),
$$

where $T^{*}$ is the adjoint of the tangle $T$ (which, by definition, is obtained from $T$ by reflecting it and moving all reflected $*$ 's clockwise by one position). Finally, the pictorial trace $\tau: P_{k} \rightarrow \mathbb{C}=P_{0_{+}}$defined by

$$
\tau(x)=\delta^{-k} Z_{t r_{k}^{0+}}^{a_{+}}(x)
$$

is postulated to be a faithful, positive, normalised trace. Here, $t r_{k}^{0_{+}}$is the tangle with a single internal $k$-box generalising $t r_{2}^{0_{+}}$of Figure 1

We illustrate the process of adjunction for tangles in an example in Figure 2 for the tangle $W$. The tangle on the left is $W$ and the dotted line is the line of 
reflection. The tangles on the right are $W^{*}$. Note that it follows that $Z_{W}(a \otimes b)^{*}=$ $Z_{W}\left(Z_{R}\left(b^{*}\right) \otimes Z_{R}\left(a^{*}\right)\right)$ in any subfactor planar algebra.
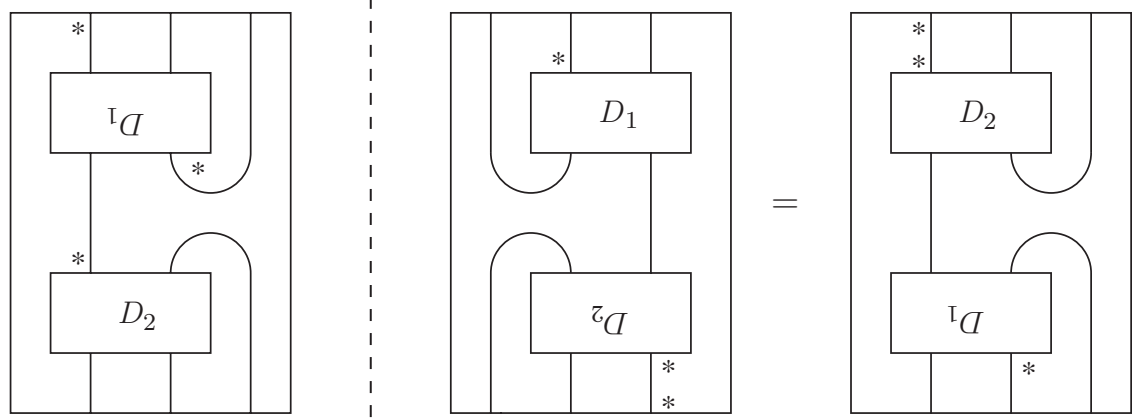

Figure 2. The adjoint of $W$

1.5. Jones' theorem. The fundamental theorem of Jones asserts that given an extremal $I I_{1}$-subfactor $N \subset M$ of finite index $\delta^{2}$ and basic construction tower $N \subset M\left(=M_{0}\right) \subset^{e_{1}} M_{1} \subset^{e_{2}} \cdots \subset^{e_{k}} M_{k} \subset^{e_{k+1}} \cdots$, there is a subfactor planar algebra $P=P^{N \subset M}$, which is unique subject to certain conditions, such that $P_{k}=$ $N^{\prime} \cap M_{k-1}$ for $k \geq 1$, and that conversely all subfactor planar algebras arise in this manner. We will use the following four consequences:

(i) the pictorial trace on $P_{k}$ agrees with the restriction to $P_{k}$ of the canonical trace on $M_{k-1}$,

(ii) for $1 \leq k \leq n, P_{k, n} \stackrel{\text { def }}{=} \operatorname{ran}\left(Z_{Q(k)_{n}^{n}}\right)$ is equal to $M_{k-1}^{\prime} \cap M_{n-1}$, where $Q(k)_{n}^{n}$ is the $n$-tangle in Figure 3, where there are $k$ strands going straight through and $k$

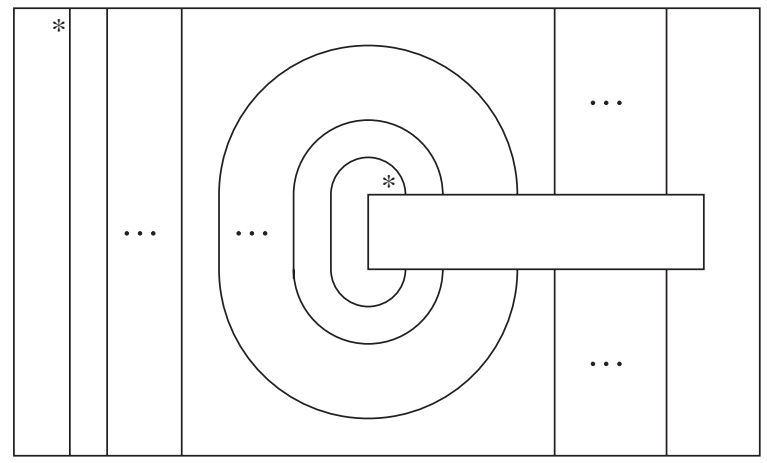

FIGURE 3 . The tangle $Q(k)_{n}^{n}$

loops on the internal $n$-box.

(iii) $Z_{\mathcal{E}^{k+1}}(1)=\delta e_{k}$ for $k \geq 1$, where $\mathcal{E}^{k+1}$ is the $k+1$-tangle generalising $\mathcal{E}^{2}$ and $\mathcal{E}^{3}$ of Figure 1, and

(iv) $Z_{\left(E^{\prime}\right)_{n}^{n}}(x)=\delta E_{M^{\prime}}(x)$ for all $x \in P_{n}$, where $E_{M^{\prime}}$ is the $N^{\prime}$-trace preserving conditional expectation of $P_{n}$ onto $M^{\prime} \cap M_{n-1}$, and $\left(E^{\prime}\right)_{n}^{n}$ is the tangle $Q(1)_{n}^{n}$. 


\section{KaC ALGEBRA STRUCTURE ON RELATIVE COMMUtANTS}

For the rest of this paper, we fix an irreducible, depth two subfactor $N \subset M$ and let $P=P^{N \subset M}$ so that $P_{0_{ \pm}}=\mathbb{C}$ and $P_{k}=N^{\prime} \cap M_{k-1}$ for $k \geq 1$. In particular, by irreducibility, $P_{1}=\mathbb{C}$. We will show, in this section, that the relative commutants $N^{\prime} \cap M_{1}$ and $M^{\prime} \cap M_{2}$ are endowed with the structure of a pair of dual Kac algebras.

2.1. Hopf and Kac algebras. We restrict ourselves to finite-dimensional algebras. Recall that a finite-dimensional complex vector space $H$ equipped with maps $\mu: H \otimes H \rightarrow H, \eta: \mathbb{C} \rightarrow H, \Delta: H \rightarrow H \otimes H, \epsilon: H \rightarrow \mathbb{C}$ and $S: H \rightarrow H$ is said to be a Hopf algebra if

(i) $(H, \mu, \eta)$ is an associative unital algebra,

(ii) $(H, \Delta, \epsilon)$ is a coassociative, counital coalgebra,

(iii) the bialgebra conditions hold, i.e., $\Delta$ and $\epsilon$ are unital algebra maps and

(iv) the antipode conditions hold, i.e., $S$ (is the unique map that) satisfies $\mu \circ$ $\left(S \otimes i d_{H}\right) \circ \Delta=\eta \circ \epsilon=\mu \circ\left(i d_{H} \otimes S\right) \circ \Delta$.

The dual vector space $H^{*}$ admits a Hopf algebra structure for the dual maps $\Delta^{*}, \epsilon^{*}, \mu^{*}, \eta^{*}$ and $S^{*}$.

A Hopf algebra $H$ is said to be a Kac algebra if it is, in addition to all the above, a $C^{*}$-algebra with $\Delta$ being a $*$-homomorphism. The dual vector space then acquires a Kac algebra structure for the $*$-structure defined by $f^{*}(a)=\overline{f\left(S a^{*}\right)}$.

We will find it convenient to adopt (with some minor changes) Sweedler's notation for comultiplication and its iterates. In this notation it is usual to denote $\Delta(a)$ by $\sum_{(a)} a_{(1)} \otimes a_{(2)}$. We will omit the parentheses from the subscripts and the summation symbol and simply denote $\Delta(a)$ by $a_{1} \otimes a_{2}$.

2.2. Definition of $S, \Delta$ and $\epsilon$. Let $S: P_{2} \rightarrow P_{2}$ be $Z_{R}$. The tangle equations $R \circ R=I_{2}^{2}$ and $R^{*}=R$ imply that $S$ is involutive (in particular, surjective) and commutes with $*$. The depth assumption implies that an arbitrary element of $P_{3}$ can be written as a linear combination of those of the form $a e_{2} b$ for $a, b \in P_{2}$, and therefore also as a linear combination of $(S a) e_{2} b=\delta^{-1} Z_{W}(a \otimes b)$, the equality following from (iii) above. This shows that $Z_{W}: P_{2} \otimes P_{2} \rightarrow P_{3}$ is surjective. Consideration of the graph invariants of $N \subset M$ in conjunction with the depth and irreducibility assumptions shows that the index $\delta^{2}$ is an integer (which we denote $n$ ) and that the dimension of $P_{k}$ is $n^{k-1}$. Hence $Z_{W}$ is an isomorphism. The equations of Figure 4 hold for each $a \in P_{2}$ since $P_{1}$ is $\mathbb{C}$ and so an arbitrary element is a scalar multiple of its identity $\mathbb{1}_{1}$, where the scalar is just its trace. The last equality appeals, in addition, to the sphericality of $P$.

For $a \in P_{2}$, define $\Delta(a) \in P_{2} \otimes P_{2}$ and $\epsilon(a) \in \mathbb{C}=P_{0_{+}}$by the equations

$$
\Delta(a)=Z_{W}^{-1} \circ Z_{F}(a), \quad \epsilon(a)=\delta^{-1} Z_{L}(a) .
$$

Figure 5 is a pictorial depiction of these definitions.

We now show that $P_{2}$ endowed with the maps $\Delta, \epsilon$ and $S$ is a Kac algebra with dual Kac algebra $P_{1,3}$.

2.3. $\Delta$ and $\epsilon$ are unital algebra maps. The multiplicativity of $\Delta$ follows from Figure [6 which shows that $Z_{F}(a b)=Z_{W}\left(a_{1} b_{1} \otimes a_{2} b_{2}\right)$.

That $\Delta$ is unital is a consequence of the obvious tangle equation $F \circ 1^{2}=$ $W \circ_{\left(D_{1}, D_{2}\right)}\left(1^{2}, 1^{2}\right)$. Multiplicativity of $\epsilon$ follows from its definition and use of Figure 4 while $\epsilon\left(\mathbb{1}_{2}\right)=1$ follows from the fact that the planar algebra $P$ has modulus $\delta$. 


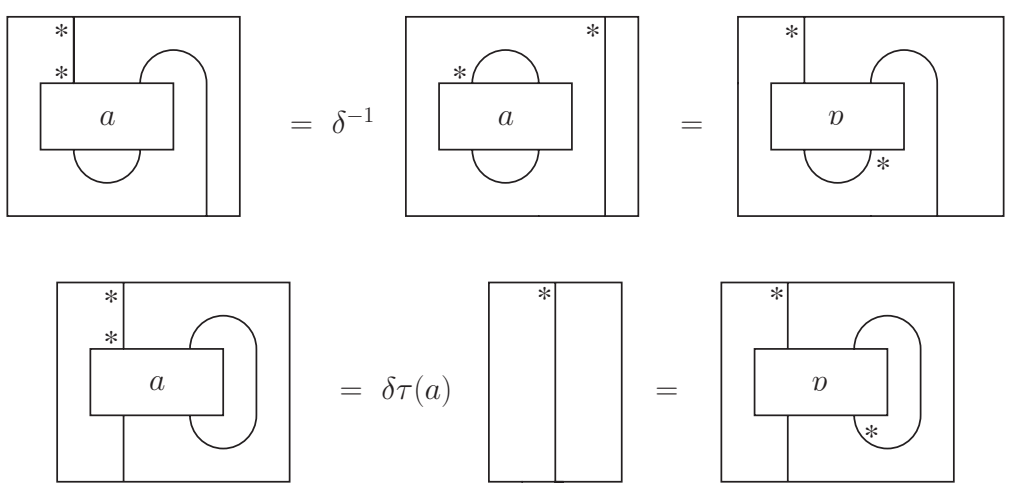

Figure 4. Pictorial equations for $a \in P_{2}$

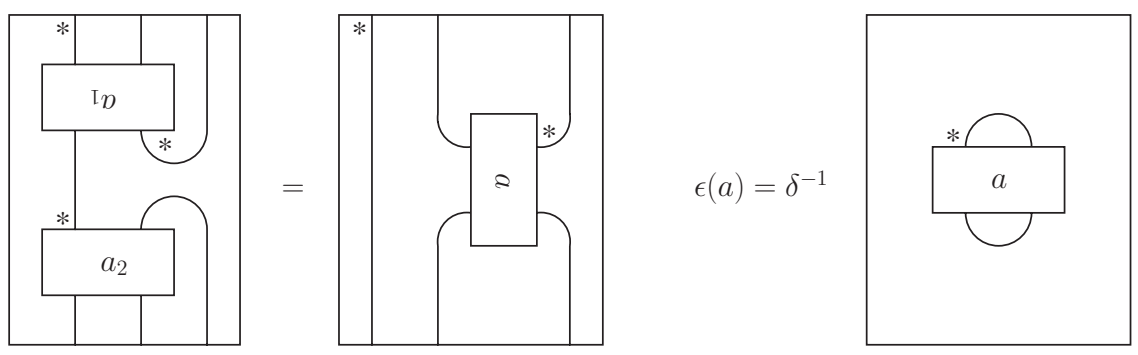

Figure 5. Pictorial definitions of $\Delta$ and $\epsilon$

2.4. Coassociativity of $\Delta$ and counitality of $\epsilon$. We prove these by showing that with respect to a non-degenerate pairing between $P_{2}$ and $P_{1,3}, \Delta$ and $\epsilon$ are dual, respectively, to the multiplication and unit of $P_{1,3}$. The tangle $B$ gives a map $Z_{B}: P_{2} \otimes P_{3} \rightarrow \mathbb{C}$. Since $Z_{F}$ is known - and easily verified - to give an isomorphism from $P_{2}$ onto $P_{1,3}$ and $Z_{B}\left(a \otimes Z_{F}(b)\right)=\delta^{2} \tau(a b)$, the non-degeneracy of $\tau$ implies that the pairing $\langle\cdot \mid \cdot\rangle: P_{2} \otimes P_{1,3} \rightarrow \mathbb{C}$ defined by $\langle a \mid f\rangle=\delta^{-1} Z_{B}(a \otimes f)$ for $a \in P_{2}$ and $f \in P_{1,3}$ is a non-degenerate pairing satisfying $\left\langle a \mid Z_{F}(b)\right\rangle=\delta \tau(a b)$.

Verifying that $P_{2}$ and $P_{1,3}$ are a dual pair of bialgebras amounts to checking that

$$
\begin{aligned}
\left\langle a_{1} \mid f\right\rangle\left\langle a_{2} \mid g\right\rangle & =\langle a \mid f g\rangle \text { and } \\
\epsilon(a) & =\left\langle a \mid \mathbb{1}_{3}\right\rangle,
\end{aligned}
$$

for all $a \in P_{2}$ and $f, g \in P_{1,3}$. We set $f=Z_{F}(x), g=Z_{F}(y)$ for $x, y \in P_{2}$. Observe that $Z_{F}(x) Z_{F}(y)=Z_{(F \circ C)}(x \otimes y)$ and therefore Figure 7 verifies the first equation. The second equation holds since $B \circ_{D_{2}} 1^{3}=L$ and by definition of $\epsilon$.

2.5. The antipode condition. To see that they are Hopf algebras, it suffices to check that $i d_{P_{2}}$ has a one-sided convolution inverse, for then the finite dimensionality implies that it is an antipode for $P_{2}$. The proof in Figure 8 shows that $S\left(a_{1}\right) a_{2}=\epsilon(a) \mathbb{1}_{2}$ for $a \in P_{2}$. Note the second equality follows from the pictorial definition of $\Delta$, while the third is a consequence of the pictorial definition of $\epsilon$ and Figure 4. Hence $P_{2}$ and $P_{1,3}$ are a dual pair of Hopf algebras. In particular, $S$ is an anti-algebra and anti-coalgebra map. 

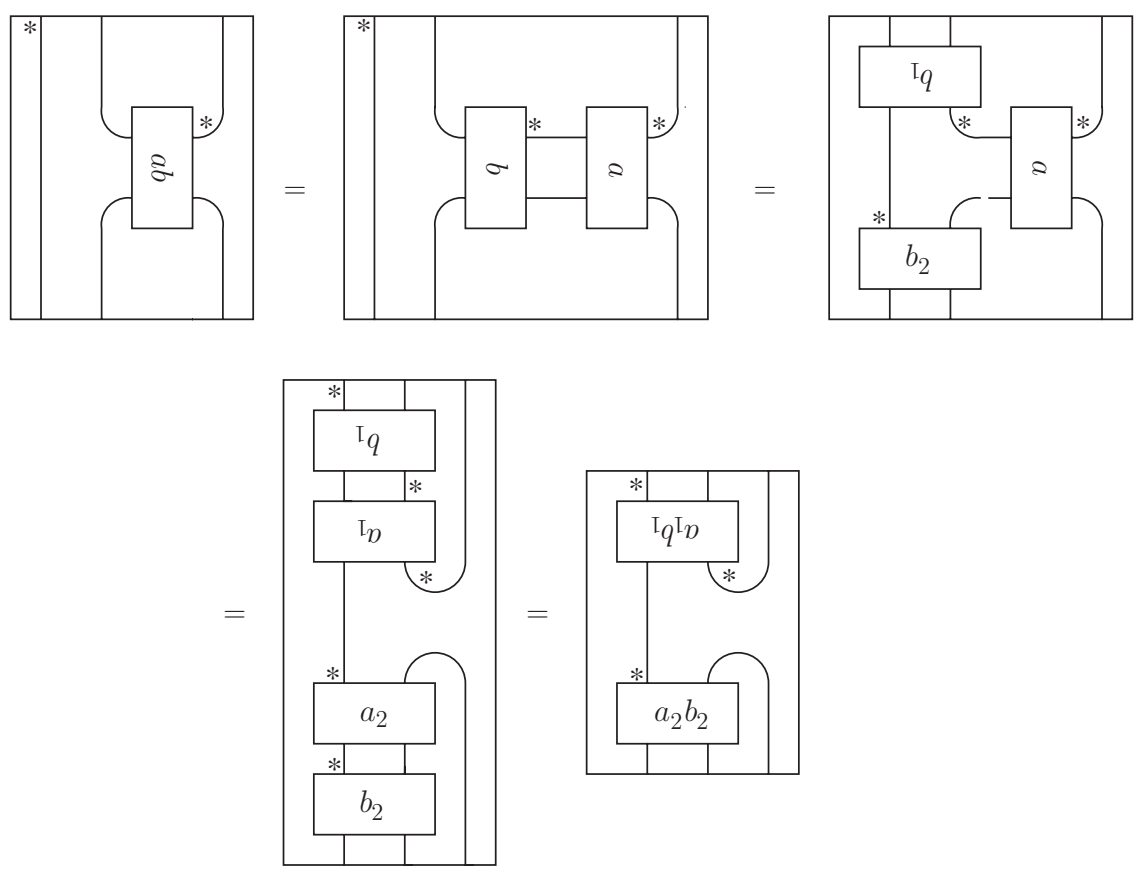

FIgURE 6 . Multiplicativity of $\Delta$
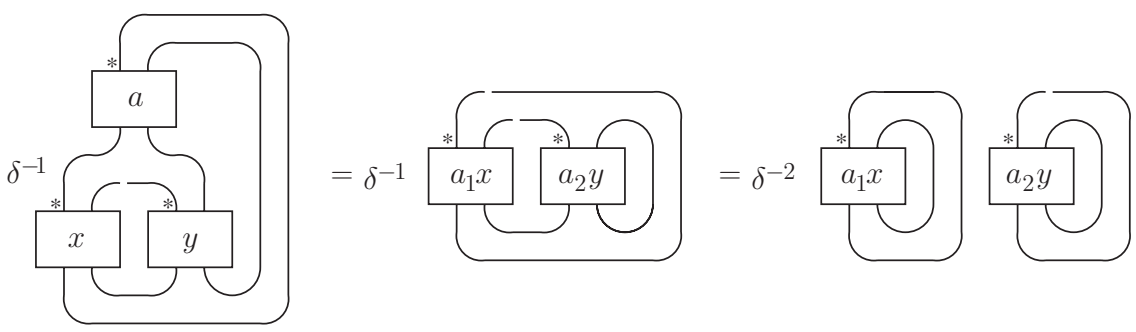

Figure 7. Duality for $\Delta$
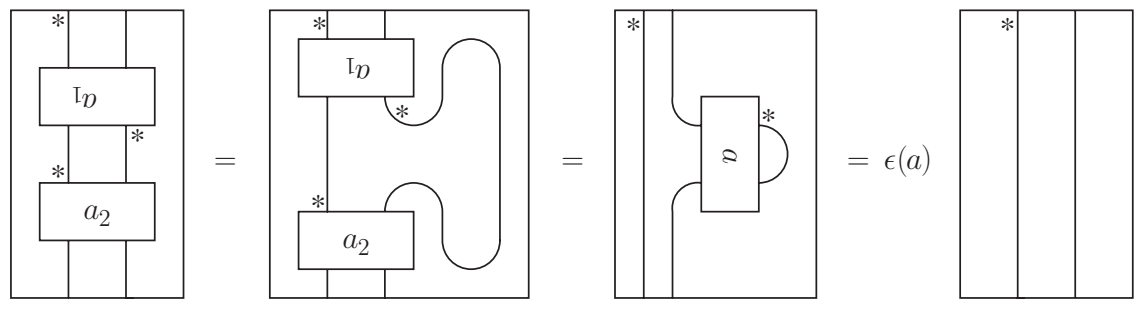

Figure $8 . S$ is an antipode

2.6. $\Delta$ is a $*$-map. To see that $\Delta$ commutes with $*$, use the definition of the adjoint tangle to take $*$ of both sides of $Z_{W}\left(a_{1} \otimes a_{2}\right)=Z_{F}(a)$. This gives

$$
Z_{W}\left(S\left(\left(a_{2}\right)^{*}\right) \otimes S\left(\left(a_{1}\right)^{*}\right)\right)=Z_{F}\left(S\left(a^{*}\right)\right) .
$$


Now use the commutativity of $S$ and $*$ in all three places, replace $S a$ by $a$, and use that $S$ is an anti-coalgebra map to read off that $a_{1}^{*} \otimes a_{2}^{*}=\left(a^{*}\right)_{1} \otimes\left(a^{*}\right)_{2}$.

2.7. Kac algebra duality. Finally, to see that $\left\langle a \mid f^{*}\right\rangle=\overline{\left\langle S a^{*} \mid f\right\rangle}$, suppose that $f=Z_{F}(b)$ for $b \in P_{2}$. It is easy to see that $f^{*}=Z_{F}\left(S b^{*}\right)$. Then $\left\langle a \mid f^{*}\right\rangle=\delta \tau\left(a S b^{*}\right)$, while $\left\langle S a^{*} \mid f\right\rangle=\delta \tau\left(S a^{*} b\right)$. These are conjugates of each other since $\tau$ is positive, $\tau \circ S=\tau$ by sphericality of $P$, and $S$ is a $*$-preserving anti-algebra involution. This establishes duality as Kac algebras.

\section{ACTION ON $M$}

We will now verify that - in the hyperfinite case - the Kac algebra $P_{2}=N^{\prime} \cap M_{1}$ 'acts on the factor $M$ with invariants $N$ '.

3.1. Definition of an anti-action. Begin by defining, for $a \in P_{2}$, a map $\alpha_{a}^{(n)}$ : $P_{1, n} \rightarrow P_{1, n}$ pictorially by the equation in Figure 9. Note that, by definition, the argument of $\alpha_{a}^{(n)}$ is an arbitrary element of $P_{1, n}$. It should be clear that the $\alpha_{a}^{(n)}$ are compatible with the inclusions of $P_{1, n}$ into $P_{1, n+1}$ and thus patch up to define a map, which we will denote $\alpha_{a}$, from $\bigcup_{n} P_{1, n}$ to itself.

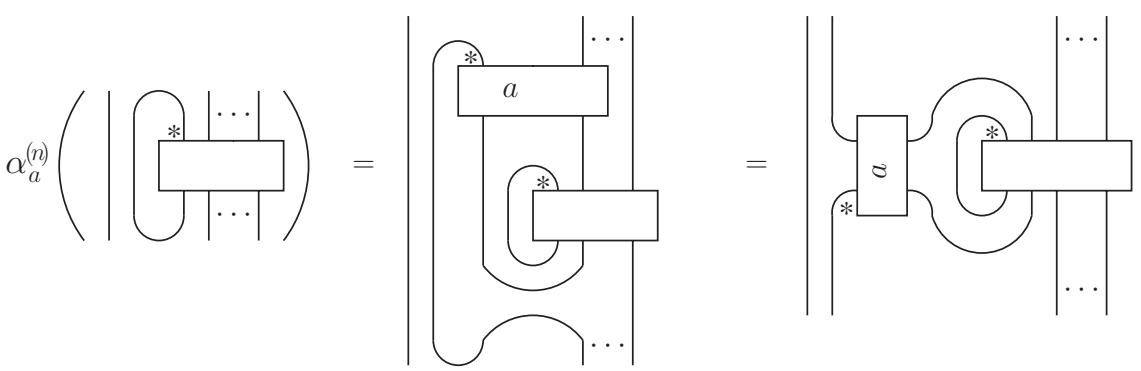

Figure 9. Definition of the action

3.2. Invariants of the anti-action. Easy pictorial proofs show that $\alpha_{1}=i d$, $\alpha_{a b}=\alpha_{a} \circ \alpha_{b}$ and that $\alpha_{a}\left(\mathbb{1}_{k}\right)=\epsilon(a) . \mathbb{1}_{k}$ for all $a, b \in P_{2}$. The two other verifications needed to see that $a \mapsto \alpha_{a}$ defines an anti-action of $P_{2}$ on $\bigcup_{n} P_{1, n}$ are that $\alpha_{a}(x y)=$ $\alpha_{a_{2}}(x) \alpha_{a_{1}}(y)$-recall the notation introduced in $\$ 2.1$ and that $\alpha_{a}(x)^{*}=\alpha_{S a^{*}}\left(x^{*}\right)$. The former follows from Figure 10 below, while the latter is an easy consequence of the definition of the adjoint tangle (and, of course, the fact that $P$ is a subfactor planar algebra).

Observe that for this anti-action of $P_{2}$ on $P_{1, n}$, the 'invariants' defined by $\{x \in$ $P_{1, n}: \alpha_{a}(x)=\epsilon(a) . x$ for all $\left.a \in P_{2}\right\}$ are exactly the elements of $P_{2, n}$. That elements of $P_{2, n}$ are invariant follows from the definition of the action and Figure 4 . For the other inclusion, observe that $\alpha_{e_{1}}\left(Z_{Q(1)_{n}^{n}}(y)\right)=Z_{Q(2)_{n}^{n}}(y)$ and therefore for $x \in P_{1, n}$, even $\alpha_{e_{1}}(x)=x$ implies that $x \in P_{2, n}$.

3.3. Conclusion of the proof. Using Jones' theorem to identify $N^{\prime} \cap M_{n-1}$ with $P_{1, n}$ and $M^{\prime} \cap M_{n-1}$ with $P_{2, n}$, we have thus defined an anti-action $x \mapsto \alpha_{a}(x)$ of $P_{2}$ on $\bigcup_{n} N^{\prime} \cap M_{n-1}$ with invariants $\bigcup_{n} M^{\prime} \cap M_{n-1}$. An appeal to consequence (iv) of Jones' theorem and the first equality in Figure 9 shows that $\alpha_{a}(x)=\delta^{2} E_{M^{\prime}}\left(a x e_{1}\right)$ and that therefore $\alpha_{a}$ is continuous for the weak topology on $N^{\prime}$. This implies that 


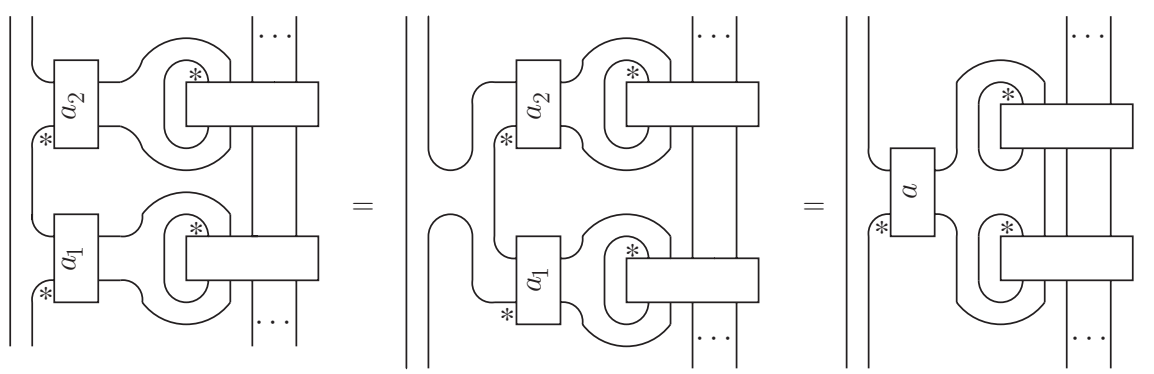

FIGURE 10. Verification of $\alpha_{a}(x y)=\alpha_{a_{2}}(x) \alpha_{a_{1}}(y)$

$\alpha_{a}$ admits a unique weakly continuous extension to the weak closure $M^{\prime} \cap M_{\infty}$ which is still an anti-action and has invariants $M_{1}^{\prime} \cap M_{\infty}$. Here $M_{\infty}$ is the weak closure of $\cup M_{n}$ in its natural representation on the Hilbert space obtained by the GNS construction with respect to its unique trace. In the hyperfinite case, the fundamental result of Popa (see Theorem 5.5.1 of $[\mathrm{Ppa}]$ ) shows that the subfactor $M_{1}^{\prime} \cap M_{\infty} \subset M^{\prime} \cap M_{\infty}$ is anti-isomorphic to $N \subset M$ and thus the anti-action of $P_{2}$ gets translated to an action on $M$ with invariants $N$.

\section{ACKNOWLEDGEMENT}

We thank V. S. Sunder for reasons that are too many to list.

\section{REFERENCES}

[BhmNllSzl] G.Bohm, F.Nill and K.Szlachanyi, Weak Hopf algebras. I. Integral theory and $C^{*}$ structure, J. Algebra 221 (1999), no. 2, 385-438 MR1726707(2001a:16059)

[Das] Paramita Das, Weak Hopf $C^{*}$-algebras and depth two subfactors, J. Funct. Anal. 214 (2004), no. 1, 74-105 MR2079886

[Jns] V. F. R. Jones, Planar Algebras I, New Zealand Journal of Mathematics, To appear.

[KdyLndSnd] V. Kodiyalam, Zeph Landau and V. S. Sunder, The planar algebra associated to a Kac algebra, Proc. Ind. Acad. Sciences, 113 (2003), no. 1, 15-51 MR 1971553 (2004d:46075)

[NksVnr] D.Nikshych, L.Vainerman, A characterization of depth 2 subfactors of $I I_{1}$ factors, J. Funct. Anal. 171 (2000), no. 2, 278-307 MR.1745634 (2000m:46129)

[Cnn] A.Ocneanu, Quantized groups, string algebras and Galois theory for algebras, Operator algebras and applications, Vol. 2, 119-172, London Math. Soc. Lecture Note Ser., 136, Cambridge Univ. Press, Cambridge, 1988. MR0996454 (91k:46068)

[Ppa] S. Popa, Classification of amenable subfactors of type II, Acta Math. 172 (1994), 163-255. MR 1278111 (95f:46105)

[Szy] W.Szymański, Finite index subfactors and Hopf algebra crossed products, Proc. Amer. Math. Soc. 120 (1994), no. 2, 519-528. MR1186139(94d:46061)

The Institute of Mathematical Sciences, Taramani, Chennai, India 600113

E-mail address: pdas@imsc.res.in

Current address: Department of Mathematics and Statistics, University of New Hampshire, Durham, New Hampshire 03824

E-mail address: pnt2@unh.edu

The Institute of Mathematical Sciences, Taramani, Chennai, India 600113

E-mail address: vijay@imsc.res.in 